\title{
The Relationship between Zika Virus and Microcephaly in Infants
}

Created by: Evangelia Antoniou ${ }^{1}$, Eirini Orovou ${ }^{2}$, Angeliki Sarella $^{3}$, Maria lliadou $^{2}$

${ }^{4}$, Nikolaos Rigas ${ }^{5}$, Ermioni Palaska ${ }^{6}$, Georgios latrakis $^{7}$, Maria Dagla ${ }^{8}$

1, University of West Attica; lilanton@uniwa.gr

2, University of West Attica; eorovou@uniwa.gr

3, University of West Attica; asare@uniwa.gr

4, Department of Midwifery, University of West Attica, Athens, Greece; miliad@uniwa.gr

5, Greek Open University; nikosstrigas@gmail.com

6, University of West Attica; epalaska@uniwa.gr

7, University of West Attica; giatrakis@uniwa.gr

8, Department of Midwifery, University of West Attica, Athens, Greece; mariadagla@uniwa.gr

Version received: 23 June 2020

check for updates

Zika virus is a newly emerging disease with little previous information related to its clinical, epidemiological and socioeconomic effects. The complexityof prevention, treatment and care to infected women and neonates, makes Zika one of the major public health problems. We provide an overview of Zika virus infection and the risk of microcephaly in the context of perinatal public health.

The present study provides strong evidence for a probable link between Zika virus infection and congenital malformations resulting in serious malformations in children. We see a clear lead in studies from Brazil and Latin America in general that the virus is in the form of an epidemic and the lack of prospective studies so far are due to the short time of the virus and microcephaly worldwide.

The results, according to the articles, show that the prevalence of microcephaly cases in Southern America (confirmed by laboratory tests), has been shown to be related to maternal infection with Zika virus in pregnancy, especially in the first and second trimesters ${ }^{[1]}$. Particularly in the first trimester, the disorder is associated with the process of neuronal migration and spinal cord formation. Of course, while the first trimester appears to be more prone to risk than the second trimester, cases of microcephaly are confirmed (or not) by reporting mothers' infection symptoms at weeks 18$20^{[2]}$. The fact that $80 \%$ of infections in pregnant women are asymptomatic, while high viral load values in the fetal brain, placenta, and fetal membranes are isolated, indicates that the timing of infection is crucial for the outcome of the disease ${ }^{[3]}$. Therefore, the risk of developing Zika virus infection is greater in the first and second trimesters of pregnancy as the CNS and brain develop. It is also of importance that Zika does not cross only the placental barrier. Elements of the virus genome were found in breast milk, saliva, urine, and mother's blood serum even a few days after childbirth 4$][5]$.

Certainly, the presence of these brain lesions is not necessary for the diagnosis of microcephaly. Some infants with the outcome did not show any brain damage during the imaging procedures presented. This suggests that congenital Zika syndrome may occur in infants with microcephaly and without the presence of imaging brain abnormalities. According to these findings, public health surveillance authorities should not disregard patients with microcephaly without findings from imaging methods $[6]$. Concerning the prevention of virus infection, so far all epidemiological studies have shown that Zika infection during pregnancy causes catastrophic neurodevelopmental effects on human embryos, but there is currently no effective treatment or prevention of Zika virus infection due to exposure to Aedes mosquitoes. Inoculation attempts in experimental animals before pregnancy, early, or mid-pregnancy, indicate fetal death or genetic abnormalities with microcephaly ${ }^{[7]}$.

Regarding preventive measures, international organizations (CDC/MEDICHEM/WHO) have made recommendations on Zika virus and microcephaly in infants. The CDC recommends measures to prevent disease and transmission. The best way to prevent is a protection with special mosquito repellent measures and the avoidance of direct contact of people with Zika virus, due to patient's body fluids as the infection is transmitted in this way ${ }^{[8]}$. Prevention in pregnancy involves appropriate family planning methods in the endemic areas, thorough parental care in affected countries, 


\section{Encyclopedia}

avoiding unnecessary trips to endemic countries, especially by people of reproductive age. Based on the available data, a woman who is not pregnant will not be at risk for birth defects in future pregnancies, as long as relative immunity has developed. According to the above recommendations, the importance of prevention by avoiding travel to endemic countries by people of both sexes is emphasized. Contraceptive measures in confirmed cases are proposed at least for a period of 6 months after infection in men. Currently there is no cure or vaccine to prevent Zika virus, the treatment is coincidental and the only way to prevent it is to prevent mosquitoes breeding in stagnant waters, insecticides, and bite protections ${ }^{[9]}$.

The World Health Organization (WHO), in response to the outbreak of Zika virus, has set out a response plan, outlining four key goals with emphasis on the prevention and management of neonatal microcephaly caused by Zika infection. These goals include detection, prevention, care support, and research. Given the current situation, the spread of Zika virus is expected to have long-term effects on health, for families, communities, and countries whose health systems will be called upon to care for children born with these complications for years to come ${ }^{[10]}$. Finally, giving the limitations of cross-sectional studies (that do not account for the incidence of the disease) and case studies (that provide a minimum basis for scientific generalization), a design of cohort studies is the most appropriate to established the relationship between Zika virus and microcephaly in infants.

\section{Conclusions}

In the present study, an attempt was made to approximate the frequency and conditions under which Zika may cause microcephaly as well as factors increasing the risk of vertical transmission in pregnancy.

Most studies have linked maternal infection during pregnancy to the appearance of brain damage in newborns. Consequently, congenital microcephaly is the hallmark of Zika's intrauterine infection. The period considered most dangerous for the vertical transmission of the infection is the first trimester and the beginning of the second or the entire second trimester, as with the Toxoplasmosis, Rubella, Cytomegalovirus and Herpes TORCH and all pathogens.

Therefore, for countries with outbreaks of Zika, it is advisable to prevent unintended pregnancies and take measures to combat transmission of Zika virus from infected mosquitoes, especially during the first and second trimesters of the nervous system development.

In order to better understand the relationship between Zika infection and neonatal microcephaly, further research will be needed, because the prevalence of Zika infection and risk of vertical transmission remains high. A cohort study of pregnant women will be able to estimate the time from onset of Zika infection and associate it with the full range of adverse pregnancy outcomes.

Zika virus epidemics have significant short and long-term impacts in the socioeconomic spheres in the Americas. In addition to the huge financial losses due to a heavy dependence on tourism and increased stress on the healthcare system, affected individuals with microcephaly may be unable to join the labor force in the future. The Zika epidemic has disproportionately affected the poorest countries, as well as the vulnerable groups creating unequal social and health service and contributing to widening inequalities in the region. As recently observed, epidemics spread by mosquitoes (Zika, yellow fever, dengue, chikungunya) can expand rapidly, including to other parts, which highlights the need for effective control of the vectors.

\section{References}

1. Driggers, R.W.; Ho, C.-Y.; Korhonen, E.M.; Kuivanen, S.; Jääskeläinen, A.J.; Smura, T.; Rosenberg, A.; Hill, D.A.; DeBiasi, R.L.; Vezina, G.; et al.et al. Zika Virus Infection with Prolonged Maternal Viremia and Fetal Brain Abnormalities. N. Engl. J. Med 2016, 374, 2142-2151, DOI: 10.1056/NEJMoa1601824.

2. Melo, A.S.d.O.; Aguiar, R.S.; Amorim, M.M.R.; Arruda, M.B.; Melo, F.d.O.; Ribeiro, S.T.C.; Batista, A.G.M.; Ferreira, T.; Dos Santos, M.P.; Sampaio, V.V.; et al.et al. Congenital Zika Virus Infection: Beyond Neonatal Microcephaly. . y. JAMA Neurol 2016, 73, 14071416, doi:10.1001/jamaneurol.2016.3720.

3. Driggers, R.W.; Ho, C.-Y.; Korhonen, E.M.; Kuivanen, S.; Jääskeläinen, A.J.; Smura, T.; Rosenberg, A.; Hill, D.A.; DeBiasi, R.L.; Vezina, G.; et al.et al Zika Virus Infection with Prolonged Maternal Viremia and Fetal Brain Abnormalities. N. Engl. J. Med. 2016, 374, 2142-2151, DOI: 10.1056/NEJMoa1601824. 
4. Pacheco, O.; Beltrán, M.; Nelson, C.A.; Valencia, D.; Tolosa, N.; Farr, S.L.; Padilla, A.V.; Tong, V.T.; Cuevas, E.L.; Espinosa-Bode, A.; et al.et al. Zika Virus Disease in Colombia_Preliminary Report. N. Engl. J. Med 2016, 0, 0, DOI: 10.1056/NEJMoa1604037.

5. Calvet, G.; Aguiar, R.S.; Melo, A.S.O.; Sampaio, S.A.; Filippis, I.d.; Fabri, A.; Araujo, E.S.M.; Sequeira, P.C.d.; Mendonça, M.C.L.d.; Oliveira, L.d.; et al.et al. Detection and Sequencing of Zika Virus from Amniotic Fluid of Fetuses with Microcephaly in Brazil: A Case Study. L. Lancet Infect. Dis 2016, 16, 653-660, /doi.org/10.1016/S1473-3099(16)00095-5.

6. CDC. Facts about Microcephaly. Birth Defects. NCBDDD . CDC. Retrieved 2020-6-11

7. Rice, M.E.; Galang, R.R.; Roth, N.M.; Ellington, S.R.; Moore, C.A.; Valencia-Prado, M.; Ellis, E.M.; Tufa, A.J.; Taulung, L.A.; Alfred, J.M.; et al.et al. Vital Signs: Zika-Associated Birth Defects and Neurodevelopmental Abnormalities Possibly Associated with Congenital Zika Virus Infection-U.S. Territories and Freely Associated States. Morb. Mortal. Wkly 2018, 67, 858-867, doi.org/10.15585/mmwr.mm6731e1..

8. Zika Viruss-Prevention and Transmission. . CDC. Retrieved 2020-6-11

9. Top 10 Facts on Zika Virus . MEDINDIA. Retrieved 2020-6-11

10. WHO. World Health Organization . WHO. Retrieved 2020-6-11

\section{Keywords}

Zika virus; Zika virus and pregnancy; Zika virus and microcephaly; Zika virus and congenital malformations.; Zika virus and fetal toxicity; Zika virus and craniostenosis; Zika virus and congenital malformations; Zika virus and teratogenesis; virus and children' 